\title{
On the Uniqueness of the ADS Spacetime
}

\author{
Xiaodong Wang * \\ September 5, 2002
}

\begin{abstract}
The uniqueness of the ADS spacetime among all static vacuum spacetimes with the same conformal infinity is proved for dimension $n \leq 7$. For dimension $n>7$, the same result is established under the spin assumption.
\end{abstract}

Keywords: Static vacuum, ADS spacetime, Asymptotically hyperbolic manifold, Positive mass theorem

\section{$\operatorname{MR}(2000)$ Subject Classification 53, 58}

In Einstein's theory of general relativity with a negative cosmological constant $\Lambda$, a vacuum spacetime is a solution to the equation $R_{a b}=\Lambda g_{a b}$ and the lowest-energy solution is the anti-de Sitter spacetime. Moreover it was proved by Bocher-GibbonsHorowitz [1] in $3+1$ dimensions that the anti-de Sitter spacetime is the unique static, asymptotically anti-de Sitter vacuum.

To give the precise statement of their theorem, let us first recall that an $(n+1)$ dimensional static spacetime $(N, \bar{g})$ has the form

$$
\begin{aligned}
N & =\mathbb{R} \times M, \\
\bar{g} & =-V^{2} d t^{2}+g,
\end{aligned}
$$

where $(M, g)$ is a Riemannian manifold and $V$ is a positive function on $M$. The vacuum Einstein equation (without loss of generality we always take the negative cosmological constant $\Lambda$ to be $-n$ )

$$
\operatorname{Ric}(\bar{g})=-n \bar{g}
$$

${ }^{*}$ Department of Mathematics, MIT, Cambridge, MA 02139, USA. Email:xwang@math.mit.edu 
can be written in terms of $g$ and $V$ as

$$
\begin{aligned}
\operatorname{Ric}(g)+n g & =V^{-1} D^{2} V, \\
\triangle V & =n V,
\end{aligned}
$$

where $D^{2}$ and $\triangle$ are the Hessian and Laplacian on $(M, g)$. From these two equations it is easy to see that $g$ has scalar curvature $R=-n(n-1)$. We will often just call the triple $(M, g, V)$ a static vacuum.

The anti-de Sitter spacetime, in $(n+1)$ dimensions, can be written as

$$
d s^{2}=-\left(1+r^{2}\right) d t^{2}+\left(1+r^{2}\right)^{-1} d r^{2}+r^{2} d \omega^{2},
$$

which is manifestly static. It is simply $\mathbb{R} \times \mathbb{H}^{n}$ with the metric $-\cosh ^{2} r d t^{2}+h$, where $h=d r^{2}+\sinh ^{2} r d \omega^{2}$ is the hyperbolic metric in polar coordinates. Another interesting example is the so called AdS soliton, which is $\mathbb{R} \times B^{2} \times T^{n-2}$ with the metric

$$
d s^{2}=-r^{2} d t^{2}+V(r)^{-1} d r^{2}+V(r) d \phi^{2}+r^{2} h,
$$

where, $h$ is a flat metric on $T^{n-2}, V(r)=r^{2}\left(1-\frac{r_{0}^{n}}{r^{n}}\right)$, with $r_{0}>0$ a constant and $\phi$ is periodic with period $4 \pi / n r_{0}$ to resolve the singularity at $r=r_{0}$. A uniqueness theorem has recently been established by Galloway-Surya-Woolgar [6]. In both examples $(M, g)$ is conformally compact. The conformal infinity is the standard sphere $S^{n-1}$ in the anti-de Sitter case and a flat torus $T^{n}$ in the AdS soliton case.

The simplest example of a spacetime which is asymptotic to the anti-de Sitter spacetime is the Schwarzschild-anti-de Sitter metric:

$$
d s_{0}^{2}=-f(r) d t^{2}+f(r)^{-1} d r^{2}+r^{2} d \omega^{2}
$$

where $f(r)=1+r^{2}-M / r^{n-2}$. In dimension $n=3$ Boucher-Gibbons-Horowitz [1] defines a spacetime to be asymptotically anti-de Sitter if outside of a spatially compact world tube the metric has the following asymptotic behavior:

$d s^{2}=d s_{0}^{2}+O\left(r^{-2}\right) d t^{2}+O\left(r^{-6}\right) d r^{2}+O(r)$ ( remaining differentials not involving $\left.d r\right)$ $+O\left(r^{-1}\right)$ ( remaining differentials involving $\left.d r\right)$,

where $d s_{0}^{2}$ is metric in (5). With this definition they proved that the only static asymptotically anti-de Sitter solution to $R_{a b}=\Lambda g_{a b}$ is the anti-de Sitter spacetime. 
There are two important ingredients in the proof. The first one is the positive mass theorem for asymptotically hyperbolic manifolds. The second ingredient is the following remarkable identity attributed to Lindblom

$$
\operatorname{div}\left(V^{-1} \nabla\left(W-W_{0}\right)\right)=\frac{1}{4} V^{3} W^{-1}|B|^{2}+\frac{3}{4} V^{-1} W^{-1}\left|\nabla\left(W-W_{0}\right)\right|^{2},
$$

where $W=|\nabla V|^{2}, W_{0}=V^{2}-1$ and $B$ is the Bach tensor. In a local frame the Bach tensor $B_{i j k}=R_{i j, k}-R_{i k, j}+\frac{1}{4} S_{j} h_{i k}-\frac{1}{4} S_{k} h_{i j}$ and its importance lies in the fact that a Riemannian 3-manifold is locally conformally flat iff its Bach tensor $B=0$. To verify the identity (7), the key point is to express $B$ in terms of $V$ and $h$ and this is possible because the curvature tensor is determined by the Ricci tensor in dimension three. To see this choose a local orthonormal frame and compute, using (3) and $S=-6$

$$
\begin{aligned}
B_{i j k} & =R_{i j, k}-R_{i k, j} \\
& =V^{-1}\left(V_{i j, k}-V_{i k, j}+V^{-2}\left(V_{j} V_{i k}-V_{k} V_{i j}\right)\right. \\
& =V^{-1} R_{j k l i} V_{l}+V^{-2}\left(V_{j} V_{i k}-V_{k} V_{i j}\right) .
\end{aligned}
$$

In dimension three, the curvature tensor $R_{j k l i}=3\left(\delta_{j i} \delta_{k l}-\delta_{j l} \delta_{k i}\right)+R_{j l} \delta_{k i}+\delta_{k l} R_{k i}-$ $R_{j i} \delta_{k l}-\delta_{j i} R_{k l}$. By using (3) again we end up with

$$
B_{i j k}=V^{-2}\left[\left(V_{j} l V_{l}-3 V V_{j}\right) \delta_{i k}-\left(V_{k} l V_{l}-3 V V_{k}\right) \delta_{i j}+2\left(V_{i k} V_{j}-V_{i j} V_{k}\right)\right] .
$$

In higher dimensions the curvature tensor is considerably more complicated with an extra piece, the Weyl tensor which is out of the reach of the field equations $(3)(4)$, therefore it is not clear at all how to generalize (7).

The main purpose of this paper is to give a different approach which is very elementary and works in any dimensions. We also use the positive mass theorem, but in place of the Lindblom identity (7) we use an elementary identity in Riemannian geometry which holds in all dimensions. Let $f$ be a function and $T$ a symmetric 2 -tensor on a Riemannian manifold $(M, g)$, then we have

$$
\left\langle T, D^{2} f\right\rangle_{g}=\operatorname{div}\left(i_{\nabla f} T\right)-\langle d f, \delta T\rangle_{g} .
$$

In a local orthonormal frame this can be written as

$$
T_{i j} f_{i j}=\left(T_{i j} f_{i}\right)_{j}-f_{i} T_{i j, j}
$$

If $\Omega \subset M$ is a compact and smooth domain, the by Stokes theorem we obtain

$$
\int_{\Omega}\left\langle T, D^{2} f\right\rangle_{g} d \mu=\int_{\partial \Omega} T(\nabla f, \nu) d A-\int_{\Omega}\langle d f, \delta T\rangle_{g} d \mu
$$


where $\nu$ is the outer unit normal of $\partial \Omega$.

An advantage of our approach is that we use conformal compactification to formulate the asymptotics (see Wang [12] and Chrusiel-Herzlich [3]), which allows more general asymptotics. Instead of having mass $M$ as just a number, there is a mass aspect $\tau$ which is a 2-tensor. Moreover the asymptotics required for the application of the positive mass theorem follow naturally from the field equations (3) (4). With Fefferman and Graham's result on conformally compact Einstein metrics the analysis is also made clean and transparent. We state our main result as

Theorem 1 Let $(M, g, V)$ be a static solution to the vacuum Einstein equation with negative cosmological constant (2). If

1. $(M, g)$ is conformally compact and the conformal boundary is $S^{n-1}$,

2. $V^{-1}$ is a defining function and $\left.V^{-2} g\right|_{S^{n-1}}$ is the standard metric on $S^{n-1}$

3. $M$ is spin,

then $(M, g, V)$ is the anti-de Sitter spacetime.

Remark. The last assumption that $M$ is spin, which is superfluous in dimension $n=3$, is needed because that is the only case the positive mass theorem for asymptotically hyperbolic manifolds is known.

Since $(M, g)$ is conformally compact with scalar curvature $R=-n(n-1)$ we can choose a defining function $r$ such that near infinity

$$
g=r^{-2}\left(d r^{2}+h_{r}\right)
$$

where $h_{r}$ is an $r$-dependent family of metrics on the conformal boundary $S^{n-1}$ with $h_{0}$ being the unit round metric (see e. g. Lemma 2.1 in Graham [5]). We choose local coordinates $x^{1}, \ldots, x^{n-1}$ on the boundary and write $h_{r}=h_{i j}(r, x) d x^{i} d x^{j}$. Moreover in view of the assumption (ii) we can assume $\left.V r\right|_{S^{n-1}} \equiv 1$.

For example it is easy to see that the anti-de Sitter spacetime can be written in this form as

$$
\begin{aligned}
& g_{0}=r^{-2}\left(d r^{2}+\left(1-r^{2} / 4\right)^{2} h_{0}\right) \\
& V=1 / r+r / 4
\end{aligned}
$$


We need to study the asymptotic expansion of $h_{r}$ and $V$ in detail. To do this one could study directly the equations (3) and (4). Instead we take advantage of the various results available for conformally compact Einstein manifolds. We recommend Graham [5] for a clear and succinct introduction.

Consider the manifold $N=S^{1} \times M$ with the metric

$$
\tilde{g}=V^{2} d \theta^{2}+g=r^{-2}\left(d r^{2}+(V r)^{2} d \theta^{2}+h_{r}\right),
$$

where $\theta$ is periodic with period $2 \pi$. By the equations $(3)(4)(N, \tilde{g})$ is conformally compact Einstein (i. e. Ric $(\tilde{g})=-n \tilde{g})$ with conformal infinity $\left(S^{1} \times S^{n-1}, d \theta^{2}+h_{0}\right)$. If $(M, g, V)$ is the anti-de Sitter spacetime, we have by (12) (13)

$$
\tilde{g}_{0}=r^{-2}\left(d r^{2}+\left(1+r^{2} / 4\right)^{2} d \theta^{2}+\left(1-r^{2} / 4\right)^{2} h_{0}\right) \text {. }
$$

Both $\tilde{g}$ and $\tilde{g}_{0}$ are conformally compact Einstein with the same conformal infinity $\left(S^{1} \times S^{n-1}, d \theta^{2}+h_{0}\right)$. By the work of Fefferman and Graham as presented in [5] (roughly speaking, in the Taylor expansion in $r$ the first $n$ terms are locally determined by the metric $h_{0}$ on the conformal boundary), we have

$$
(V r)^{2} d \theta^{2}+h_{r}=\left(1+r^{2} / 4\right)^{2} d \theta^{2}+\left(1-r^{2} / 4\right)^{2} h_{0}+r^{n}\left(\alpha d \theta^{2}+\tau\right)+o\left(r^{n}\right),
$$

where $\tau$ is a symmetric 2-tensor on $S^{n-1}$ and

$$
\alpha=-\operatorname{tr}_{h_{0}} \tau
$$

The equation (14) can be separated as two equations

$$
\begin{aligned}
V & =1 / r+r / 4+\alpha r^{n-1} / 2+o\left(r^{n-1}\right) \\
h_{r} & =\left(1-r^{2} / 4\right)^{2} h_{0}+\tau r^{n}+o\left(r^{n}\right) .
\end{aligned}
$$

These asymptotic expansions can be differentiated. For example by differentiating (16) in $r$ we obtain

$$
\frac{\partial V}{\partial r}=-1 / r^{2}+1 / 4+(n-1) \alpha r^{n-2} / 2+o\left(r^{n-2}\right)
$$

The equation (17) shows that $(M, g)$ is asymptotically hyperbolic in the sense of $[12]$.

Definition 1 [12] A conformally compact manifold $(X, g)$ is called asymptotically hyperbolic if it satisfies: 
1. the conformal infinity is the standard sphere $\left(S^{n-1}, h_{0}\right)$,

2. there exists a good defining function $r$ such that

$$
g=r^{-2}\left(d r^{2}+h_{r}\right)
$$

in a collar neighborhood of the conformal infinity and

$$
h_{r}=\left(1-r^{2} / 4\right)^{2} h_{0}+\tau r^{n}+o\left(r^{n}\right),
$$

where $\tau$ is a symmetric 2-tenor on $S^{n-1}$. Moreover the asymptotic expansion can be differentiated twice.

For asymptotically hyperbolic spin manifolds, the following positive mass theorem was proved in Wang [12] (see also Chrusciel-Herzlich [3]).

Theorem 2 [12] Let $(X, g)$ be an asymptotically hyperbolic manifold. If $X$ is spin and has scalar curvature $R \geq-n(n-1)$ then we have

$$
\int_{S^{n-1}} \operatorname{tr}_{h_{0}}(\tau) d \mu_{h_{0}} \geq\left|\int_{S^{n-1}} \operatorname{tr}_{h_{0}}(\tau) x d \mu_{h_{0}}\right|
$$

Moreover equality holds iff $(X, g)$ is isometric to the hyperbolic space $\mathbb{H}^{n}$.

We use (16)(17) and (11) to compute

$$
\begin{aligned}
|\nabla V|^{2}-V^{2}+1= & r^{2}\left[\left(\frac{\partial V}{\partial r}\right)^{2}+h^{i j} \frac{\partial V}{\partial x^{i}} \frac{\partial V}{\partial x^{j}}\right]-V^{2}+1 \\
= & r^{2}\left[\left(-1 / r^{2}+1 / 4+(n-1) \alpha r^{n-2} / 2+o\left(r^{n-2}\right)\right)^{2}+O\left(r^{2(n-1)}\right)\right] \\
& -\left(1 / r+r / 4+\alpha r^{n-1} / 2+o\left(r^{n-1}\right)\right)^{2}+1 \\
= & -n \alpha r^{n-2}+o\left(r^{n-2}\right) .
\end{aligned}
$$

To summarize we have shown

$$
|\nabla V|^{2}-V^{2}=-1-n \alpha r^{n-2}+o\left(r^{n-2}\right)
$$

Differentiating in $r$ gives

$$
\frac{\partial}{\partial r}\left(|\nabla V|^{2}-V^{2}\right)=-n(n-2) \alpha r^{n-3}+o\left(r^{n-3}\right)
$$


Proof of the Theorem: Define $M^{\epsilon}=\{p \in M \mid r(p) \geq \epsilon\}$. For $\epsilon$ small, this is a compact and smooth domain in $M$. Let $T=\operatorname{Ric}(g)+(n-1) g$ which is actually the Einstein tensor of $g$. By (4) we have

$$
T=V^{-1} D^{2} V-g
$$

Hence $V|T|^{2}=\left\langle D^{2} V-V g, T\right\rangle_{g}=\left\langle D^{2} V, T\right\rangle_{g}$. By the second Bianchi identity $\delta T=$ $\left(\frac{1}{2}-\frac{1}{n}\right) d S=0$ for $S=-n(n-1)$. Therefore by the formula (10)

$$
\int_{M^{\epsilon}} V|T|_{g}^{2} d \mu_{g}=\int_{\partial M^{\epsilon}} T(\nabla V, \nu) d \sigma
$$

The outer unit normal $\nu=-r \frac{\partial}{\partial r}$ and the induced area form $d \sigma=r^{-(n-1)} \sqrt{H(r, x)} d x$ with $H(r, x)=\operatorname{det}\left(h_{i j}(r, x)\right)$. Therefore, using (23) again, we can rewrite the above equation as

$$
\begin{aligned}
\int_{M^{\epsilon}} V|T|_{g}^{2} d \mu_{g} & =-\epsilon^{-(n-2)} \int_{S^{n-1}}\left(V^{-1} D^{2} V\left(\nabla V, \frac{\partial}{\partial r}\right)-\left\langle\nabla V, \frac{\partial}{\partial r}\right\rangle\right) \sqrt{H(\epsilon, x)} d x \\
& =-\frac{1}{2} \epsilon^{-(n-2)} \int_{S^{n-1}} V^{-1} \frac{\partial}{\partial r}\left(|\nabla V|^{2}-V^{2}\right) \sqrt{H(\epsilon, x)} d x \\
& =\frac{n(n-2)}{2} \int_{S^{n-1}} \alpha d \sigma_{h_{0}}+o(1),
\end{aligned}
$$

where in the last step we use the asymptotic expansions (16) and (22). Taking $\epsilon \rightarrow 0$, in view of (15), we obtain

$$
\int_{M} V|T|_{g}^{2} d \mu_{g}=-\frac{n(n-2)}{2} \int_{S^{n-1}} \operatorname{tr}_{h_{0}}(\tau) d A_{h_{0}} .
$$

But by Theorem 2, the right hand side is non-positive. Therefore both sides must be zero. By the characterization of zero mass case in Theorem $2,(M, g)$ is the hyperbolic space $\mathbb{H}^{n}$. Then it is an easy matter to show that the triple $(M, g, V)$ is the anti-de Sitter spacetime.

In the last step we only need the positive mass theorem to the extent that the mass is nonnegative and the characterization of zero mass case can be replaced by an elementary argument. In fact we have $T=0$, i. e. $\operatorname{Ric}(g)+(n-1) g=V^{-1}\left(D^{2} V-\right.$ $V g)=0$. This implies that $\bar{g}=V^{-2} g$ is Einstein. Therefore $(\bar{M}, \bar{g})$ is a compact Einstein manifold with a totally geodesic boundary which is the standard sphere $S^{n-1}$. Moreover $\bar{\nabla} V$ is a conformal vector field. It is then elementary to show that $(\bar{M}, \bar{g})$ 
is the standard hemisphere $S_{+}^{n} \subset R^{n+1}$ and $V(x)=1 / x_{n+1}$. This easily implies that $(M, g, V)$ is the anti-de Sitter spacetime.

Acknowledgment: I am indebted to Professor Galloway for stimulating conversations, from whom I learned this problem during the AIM-Stanford workshop on general relativity in May, 2002. I thank the workshop organizers, especially Professor Rick Schoen, for inviting me and for providing an excellent environment. I also wish to thank Professor Tian for constant encouragement.

After the paper was submitted, the paper Qing [10] appeared. Inspired by his idea, I give an alternative approach which does not require the spin assumption in dimension $n \leq 7$. It is interesting to note that this new approach uses the positive mass theorem for asymptotically flat manifolds while the previous approach uses the positive mass theorem for asymptotically hyperbolic manifolds.

Let $(M, g, V)$ be a static AdS vacuum spacetime. By the equations (3) (4) and the Bochner formula we compute

$$
\begin{aligned}
\frac{1}{2} \triangle\left(|\nabla V|^{2}-V^{2}\right) & =\left|D^{2} V\right|^{2}+\nabla V \cdot \nabla \triangle V+\operatorname{Ric}(\nabla V, \nabla V)-V \triangle V-|\nabla V|^{2} \\
& =\left|D^{2} V\right|^{2}+V^{-1} D^{2} V(\nabla V, \nabla V)-n V^{2}-|\nabla V|^{2} \\
& =\left|D^{2} V-V g\right|^{2}-\frac{1}{2} V^{-1} \nabla V \cdot \nabla\left(|\nabla V|^{2}-V^{2}\right) .
\end{aligned}
$$

This can be rewritten as

$$
\triangle\left(|\nabla V|^{2}-V^{2}+1\right)-V^{-1} \nabla V \cdot \nabla\left(|\nabla V|^{2}-V^{2}+1\right)=2\left|D^{2} V+V g\right|^{2} \geq 0 .
$$

Suppose $(M, g, V)$ further satisfies conditions 1 and 2 in Theorem 1 . We consider the compact manifold with boundary $\bar{M}$ with the Fermat metric $\bar{g}=(V+1)^{-2} g$.

Lemma $1(\bar{M}, \bar{g})$ has scalar curvature $\bar{R} \geq 0$. Moreover the boundary is isometric to the standard sphere $S^{n-1}$ and has mean curvature $n-1$.

Proof. By (21) we have

$$
|\nabla V|^{2}-V^{2}+1 \rightarrow 0, \quad \text { as } x \rightarrow \Sigma
$$


By the maximum principle for the equation (25)

$$
|\nabla V|^{2}-V^{2}+1 \leq 0
$$

on $\bar{M}$. The scalar curvature of $\bar{R}$ of $\bar{g}$ is given by

$$
(V+1)^{-2} \bar{R}=R+2(n-1) \frac{\triangle V}{V+1}-n(n-1) \frac{|\nabla V|^{2}}{(V+1)^{2}}
$$

By $(26)$ and the fact $R=-n(n-1)$

$$
(V+1)^{-2} \bar{R} \geq-n(n-1)+2 n(n-1) \frac{V}{V+1}-n(n-1) \frac{V^{2}-1}{(V+1)^{2}}=0
$$

By the asymptotic expansions (17)(16) for $g$ and $V$, we can write $\bar{g}=\phi^{-2}\left(d r^{2}+h_{r}\right)$ near the boundary with $\phi=r(V+1)=1+r+r^{2} / 4+\ldots$. Then it's obvious that $\left.\bar{g}\right|_{\Sigma}=h_{0}$ is the round metric on $S^{n-1}$ and $\Sigma$ has mean curvature $\bar{H}=n-1$.

We now give another proof of Theorem 1 which does not require the spin assumption for $n \leq 7$, i. e. we have

Theorem 3 Let $(M, g, V)$ be a static solution to the vacuum Einstein equation with negative cosmological constant (2). If

1. $(M, g)$ is conformally compact and the conformal boundary is $S^{n-1}$,

2. $V^{-1}$ is a defining function and $\left.V^{-2} g\right|_{S^{n-1}}$ is the standard metric on $S^{n-1}$

3. either $n \leq 7$ or $M$ is spin,

then $(M, g, V)$ is the anti-de Sitter spacetime.

Proof. By Lemma $1,\left(\bar{M}, \bar{g}=(1+V)^{-2} g\right)$ is a compact manifold with boundary and the boundary is the standard sphere $S^{n-1}$ with mean curvature $n-1$. Then $(\bar{M}, \bar{g})$ is isometric to the unit ball $\bar{B} \subset \mathbb{R}^{n}$. To see this, we attach $\mathbb{R}^{n}-\bar{B}$ to $(\bar{M}, \bar{g})$ along the boundary and get a complete manifold with a flat end. By the asymptotic expansion for $h_{r}$ it is easy to see that the metric is at least $C^{2}$. Positive mass theorem for asymptotically manifolds then implies that it is isometric to $\mathbb{R}^{n}$ if either $n \leq 7$ or $M$ is spin. We can even weaken the regularity assumption on $g$ as long as it guarantees that the gluing is $C^{1}$ along the common boundary hypersurface. It was known to many experts that the positive mass theorem holds in this general setting and in fact 
this fact was used by Bunting and Masood-ul-alam $[2,9,8]$ to prove uniqueness for various blackhole solutions. But there had been no detailed proofs in the literature until two recent papers by Miao [7] who uses a mollification argument to reduce it to the regular case and by Shi-Tam [11] generalizing Witten's spinor argument.

Therefore $(\bar{M}, \bar{g})$ is isometric to $\bar{B}$ and in particular $\bar{R}=0$. By the proof of Lemma 1, we have $D^{2} V=V g$ and $|\nabla V|^{2}-V^{2}+1=0$. Then by (3) we have $\operatorname{Ric}(g)=-(n-1) g$. As $g$ is also conformally flat, it follows that $(M, g)$ is the hyperbolic space.

\section{References}

[1] W. Boucher, G. W. Gibbons, and Gary T. Horowitz, Uniqueness theorem for anti-de Sitter spacetime, Phys. Rev. D (3) 30 (1984), no. 12, 2447-2451. MR $86 \mathrm{e}: 83014$

[2] Gary L. Bunting and A. K. M. Masood-ul Alam, Nonexistence of multiple black holes in asymptotically Euclidean static vacuum space-time, Gen. Relativity Gravitation 19 (1987), no. 2, 147-154. MR 88e:83031

[3] Piotr T. Chruściel and Marc Herzlich, The mass of asymptotically hyperbolic riemannian manifolds, e-Print math.DG/0110035.

[4] Piotr T. Chruściel and Walter Simon, Towards the classification of static vacuum spacetimes with negative cosmological constant, J. Math. Phys. 42 (2001), no. 4, 1779-1817. MR 1820431

[5] C. Robin Graham, Volume and area renormalizations for conformally compact Einstein metrics, The Proceedings of the 19th Winter School "Geometry and Physics" (Srní, 1999), no. 63, 2000, pp. 31-42. MR 2002c:53073

[6] G.J. Galloway, S. Surya, and E. Woolgar, On the geometry and mass of static, asymptotically ads spacetimes, and the uniqueness of the ads soliton, e-Print hep-th/0204081.

[7] Pengzi Miao, Positive Mass Theorem on Manifolds admitting Corners along a Hypersurface, arXiv math-ph/0212025.

[8] A. K. M. Masood-ul Alam, Uniqueness proof of static charged black holes revisited, Classical Quantum Gravity 9 (1992), no. 5, L53-L55. MR 93f:83059 
[9] _ Uniqueness of a static charged dilaton black hole, Classical Quantum Gravity 10 (1993), no. 12, 2649-2656. MR 94k:83050

[10] Jie Qing, On the rigidity for conformally compact Einstein manifolds, Int. Math. Res. Not. (2003), no. 21, 1141-1153. MR 1962123

[11] Y. Shi and L. Tam, Positive mass theorem and the boundary behavior of compact manifolds with nonegative scalar curvature, arXiv math.DG/0301047.

[12] Xiaodong Wang, The mass of asymptotically hyperbolic manifolds, J. Differential Geom. 57 (2001), no. 2, 273-299. MR 2003c:53044

[13]__ On conformally compact Einstein manifolds, Math. Res. Lett. 8 (2001), no. 5-6, 671-688. MR 2003d:53075 